\title{
The effect of tumor location on overall survival for pT2-4 bladder and upper tract urothelial carcinoma following radical surgery
}

\author{
Andrew W. Tam, MD; Christine Liaw, MD; Eric Li; Andrew B. Katims, MD; \\ Rollin K. Say, MD; Zeynep Gul, MD; Jared S. Winoker, MD; Alberto Martini, MD; \\ Francois Audenet; John P. Sfakianos, MD \\ Department of Urology, Icahn School of Medicine at Mount Sinai, New York, NY, United States
}

Cite as: Tam AW, Liaw C, Li E, et al. The effect of tumor location on overall survival for pT2-4 bladder and upper tract urothelial carcinoma following radical surgery. Can Urol Assoc J 2020 October 27; Epub ahead of print. http://dx.doi.org/10.5489/cuaj.6698

Published online October 27, 2020

$* * *$

\section{Abstract}

Introduction: Historically, staging and treatment for upper tract urothelial carcinoma were extrapolated from bladder urothelial carcinoma literature. However, embryological, genetic, and anatomical differences exist between them. We sought to explore the relationship between location of urothelial cancer and overall survival (OS).

Methods: Data was culled from the National Cancer Database from 2004-2015. Patients with pT2-pT4 treated with definitive surgery were included; those with metastatic disease or who received neoadjuvant or adjuvant treatment were excluded. Patients were stratified by tumor location and pathological stage. The primary outcome was OS. Secondary outcomes were predictors of mortality in each pT stage stratum.

Results: A total of 11330 patients with bladder, 954 patients with ureteral, and 1943 patients with renal pelvis urothelial carcinoma were analyzed. Mean followup was 43.3, 39.4, and 41.4 months for bladder, ureteral, and renal pelvis, respectively. On univariable analysis, ureteral pT2 was associated with worse OS compared to both bladder (61.3 vs. 80.4 months, $p=0.007)$ and renal pelvis (61.3 vs. 80.5 months, $\mathrm{p}=0.014)$. Renal pelvis pT3 was associated with improved OS compared to both bladder (42.5 vs. 28.6 months, $\mathrm{p}=0.003)$ and ureteral (42.5 vs. 25.7 months, $\mathrm{p}<0.001)$. Renal pelvis $\mathrm{pT} 4$ had decreased survival compared to bladder (11.4 vs. 17.7 months, $\mathrm{p}<0.001)$. On multivariable Cox regression, only renal pelvis pT3 was associated with a $20 \%$ decreased risk of mortality compared to bladder pT3 (hazard ratio $0.80,95 \%$ confidence interval $0.72-0.88, \mathrm{p}<0.001)$. 
Conclusions: Renal pelvis pT3 is associated with lower mortality. Mutational and embryological differences may play a role in this disparity.

\section{Introduction}

Carcinoma of the upper tract accounts for a small minority of urothelial carcinoma (UC) - the incidence is approximately $5-10 \%$ of newly diagnosed cases. Similar to other orphan diseases, there is a relative paucity of data on upper tract urothelial carcinoma (UTUC). As a result, clinical decision-making for UTUC has been largely extrapolated from the existing literature on $\mathrm{UC}$ of the bladder. This is reflected in the bundling of guidelines for bladder and upper tract UC by both the National Comprehensive Cancer Network and the American Urological Association. Presently, the European Association of Urology is the only urological association with guideline statements tailored specifically to UTUC. ${ }^{1}$

Tumor stage and grade are widely accepted as predictors of oncological outcome with contemporary studies suggesting that differences in UTUC outcomes are attributable to these factors. ${ }^{1,2,3}$ Recently, several publications have disputed that location has an impact on prognosis. ${ }^{4-10}$ Nevertheless, genetic analyses have shown differences in the mutational profile between UTUC and bladder cancer, suggesting differences in the nature of the urothelium between the upper tract and bladder. ${ }^{11-13}$ In fact, the progenitors of bladder and upper tract urothelium are entirely different - bladder is derived from mesoderm whereas the upper tract is derived from endoderm. ${ }^{1}$ We hypothesize that this foundational difference leads to heterogeneous urothelium. We thus used the National Cancer Database (NCDB) to determine the prognostic significance of UC location on survival.

\section{Methods}

\section{Patient population}

The NCDB is a nationwide database comprised of patient-related, tumor-related, and treatment outcomes information for patients treated at Commission on Cancer accredited programs. It captures approximately $70 \%$ of all newly diagnosed cancers in the US. From 2004 to 2015, there were 59,642 patients with UTUC and 525,323 patients with bladder cancer. We included patients with AJCC pT2-pT4 bladder and UTUC who underwent open or minimally invasive radical cystectomy or radical nephroureterectomy (with bladder cuff excision) with or without lymph node dissection. Patients with prior non-urothelial malignancy, more than two urothelial tumors, more than one recurrence, variant histology (i.e. non-urothelial cell), clinically or pathologically positive nodes, and clinical or pathological metastatic disease were excluded. In order to better study the impact of embryologic and genetic differences between upper and lower tract disease, patients who received neo-adjuvant or adjuvant chemotherapy, post-operative intravesical 
chemotherapy, radiotherapy, immunotherapy, or enrolled in a clinical trial were also excluded. The justification for this being that use of these therapies in UTUC is not standard of care. Data points with missing variables for staging and treatments received were excluded. This is summarized via CONSORT diagram in figure 1. The NCDB contains de-identified patient information and therefore is exempt from internal review board approval.

\section{Study outcomes}

The database includes demographics, tumor pathology, treatments received, length of follow-up, and living/death status. Patients were stratified according to tumor location as follows: renal pelvis, ureteral, or bladder. The primary outcome was overall survival. We also sought to identify potential predictors of mortality in each pathological $\mathrm{T}$ stage stratum.

\section{Statistical analysis}

Univariable analysis of categorical data was completed with chi-squared tests, whereas continuous, non-parametric data was evaluated with Kruskall-Wallis tests. Kaplan-Meier survival curves were used to depict overall survival, and were stratified by pathological T stage. Log-rank testing was used to determine statistical significance. Predictors of overall survival were estimated with multivariable Cox proportional hazard regression. Covariates included in the regression were: age, gender, race, Hispanic origin, median income quartile $(<\$ 38,000, \$ 38,000$ $\$ 47,999, \$ 48,000-\$ 62,999$, or $\geq \$ 63,000)$, Charlson/Deyo score $(0,1$, or $\geq 2)$, era of diagnosis (2004-2006, 2007-2009, 2010-2012, or 2013-2015), facility type (community cancer program, comprehensive community cancer program, academic program, or integrated network cancer program), ICD-O-3 tumor grade, tumor size, location, nodes examined, margin status, days from diagnosis to definitive surgery, days from surgery to discharge, and readmission within 30 days of surgery. Data points with missing variables are automatically excluded from Cox regression analysis.

Statistical analysis was performed using SPSS version 25 (IBM, Armonk, NY). Reported $\mathrm{p}$-values are two-sided with statistical significance set at $\mathrm{p}<0.05$.

\section{Results}

\section{Descriptive statistics}

A total of 11,330 patients with bladder (BUC), 954 patients with ureteral (UUC), and 1,943 patients with renal pelvis (RPUC) were analyzed. The demographic data is summarized in Table 1. Mean follow-up was 43.3, 39.4, and 41.4 months for bladder, ureteral and renal pelvis respectively. The median ages for UUC and RPUC were both 74 years while that for BUC was 70 years $(\mathrm{p}<0.001)$. The proportion of females that were affected by disease differed by location $-24.1 \%$ of BUC, $43.5 \%$ of UUC, and $44.8 \%$ of RPUC $(\mathrm{p}<0.001)$. There was no difference in Charlson-Deyo scores between the three groups $(\mathrm{p}=0.605)$. Patients with RPUC presented more frequently with pT3 disease (67.7\%) compared to BUC and UUC (38.2\% and 46.1\%, 
respectively; $\mathrm{p}<0.001)$. A greater proportion of BUC had IOC-O-3 grade 3 and 4 disease $(54.5 \%$ and $40.8 \%$, respectively) compared to UUC (51.6\% and $33.1 \%$, respectively), and RPUC (48.8\% and $33.6 \%$, respectively) ( $\mathrm{p}<0.001)$. There was a greater delay to surgery for BUC versus UTUC. The median number of days to surgery was 47 (IQR 29-70) for BUC, 26 (3.5-46) for UUC, and $21(0-40)$ for RPUC $(\mathrm{p}<0.001)$.

Convalescence time was longest for BUC patients. The number of days from surgery to discharge for BUC was 8 (IQR 6-11), versus 5 (IQR 3-7) for UUC and 5 (IQR 3-7) for RPUC $(p<0.001)$. Similarly, patients with BUC had a higher rate of 30 -day readmission $(11 \%)$ compared to those with UUC or RPUC (4.9\% and 5.1\%, respectively; $p<0.001)$. 30-day mortality was statistically similar between the three groups $(p=0.105)$, though 90 -day mortality was significantly worse in both BUC and RPUC (7.5\% and $6.3 \%$, respectively) versus UUC $(5.6 \%, \mathrm{p}=0.001)$.

\section{Survival analysis}

The Kaplan-Meier curve for overall survival of all patients, stratified by location, is shown in Figure 2. There was a significant difference in overall survival between BUC and UUC $(\mathrm{p}<0.001)$, and UUC and RPUC ( $\mathrm{p}=0.028)$. Median survival was 49.2 months (95\% CI 47.051.4) for BUC, 39.2 months (95\% CI 34.2-44.2) for UUC, and 46.5 (95\% CI 42.3-50.6) for RPUC (Supplemental Table 1). Kaplan-Meier functions were also stratified by pT stage (figures 3a-c). Figure 3 a shows that overall survival was worse for pT2 UUC compared to both BUC (61.3 versus 80.4 months; $p=0.007$ ) and RPUC (61.3 versus 80.5 months, $p=0.014)$. For $p T 3$, overall survival was greater for RPUC compared to both BUC (42.5 versus 28.6 months $\mathrm{p}<0.001)$ and UUC (42.5 versus 25.7 months, $\mathrm{p}<0.001)$. BUC also displayed a survival advantage over UUC (28.6 versus 25.7 months, $\mathrm{p}=0.003$. For $\mathrm{pT} 4$, BUC was associated with greater overall survival than RPUC (17.7 versus 11.4 months, respectively; $p<0.001)$. Comparison of BUC versus UUC did not reach statistical significance $(p=0.847)$.

Multivariable Cox proportional hazards regression, stratified by $\mathrm{pT}$ stage, is summarized in Tables 2a-c. Renal pelvis location was associated with a $20 \%$ decreased risk of mortality for pT3 disease (HR 0.80, 95\% CI 0.72-0.88, p<0.001). Location was not a predictor of mortality for either pT2 or pT4 disease. For all patients, regardless of pT stage, increasing number of nodes obtained for pathological diagnosis was associated with a decreased risk of mortality, while a positive surgical margin was assocated with an increased risk of mortality. Increasing tumor size and days from surgery to discharge were weak risk factors for mortality. 


\section{Discussion}

To date, there have been multiple outcome comparisons between UTUC and bladder carcinoma with conflicting results. Several studies, including a comprehensive literature review, suggest that the differences in outcomes are attributed to tumor stage and grade rather than on location itself. ${ }^{2,4,8-13}$ Nevertheless, the EAU guidelines conclude that when controlling for stage, patients with ureteral tumors have a worse prognosis than those with renal tumors. ${ }^{14}$ Our findings further support their statement.

We excluded patients who received any type of neo-adjuvant and adjuvant therapy, due to the low usage rate for UTUC, and patients with evidence of metastatic disease. Ureteral location was associated with worse overall survival for both pT2 and pT3 disease on univariable analysis. This may be attributable to the high rate of positive surgical margins for UUC. When controlling for surgical margins on multivariable Cox regression, ureteral location was not a predictor of mortality. However, renal pelvis location was associated with improved median overall survival and decreased risk of mortality compared to both bladder and ureteral location for pT3 disease. When examining the AJCC grading system used to stage upper tract tumors, invasion of peri-pelvic fat and renal parenchyma are classified as pT3 - this closely mirrors that for bladder disease, though the prognostic significance may differ when surrounding fat or surrounding organs are involved. We postulate that benefits of renal pelvis location may be attributed to the bulk of renal parenchyma shielding from invasion of surrounding tissues. Notably, Park et al. reported that peri-pelvic fat invasion was associated with a 3.47 hazard ratio for cancer specific survival compared with renal parenchymal invasion in a retrospective review. ${ }^{15}$ The NCDB data set does not allow for determination of peri-pelvic or renal parenchymal invasion for pT3. Other studies have reported the protective effect of renal pelvis location, including a large multicenter study by Ouzzane et al. and a small single-surgeon study by Akdogan et al. ${ }^{16,17}$ These studies did not stratify by tumor pathological stage, however.

The largest study comparing bladder and UTUC found that location had an impact on survival only for pT4 disease. Rink et al. completed a multicenter retrospective study with 4,335 BUC, 877 UUC, and 1,615 RPUC patients treated with radical surgery. They reported no differences in cancer-specific survival for pT2-pT3 but worse survival for pT4 in the upper tract. $^{8}$ In contrast, we found that only pT4 RPUC did worse than BUC, though RPUC was not a predictor of mortality on Cox regression $(p=0.108)$. The inclusion of patients with positive nodes, who received adjuvant chemotherapy, and lack of surgical margin status may explain this disparity. Interestingly, their Kaplan-Meier survival curve for pT3 showed that renal pelvis location had improved overall survival when compared to both bladder and ureter, but they did not include a Cox regression. ${ }^{8}$ Moschini et al. compared survival in patients with unresectable, $\mathrm{pN}+$, and metastatic UTUC and bladder cancer treated with chemotherapy and reported no significant differences in survival when stratified by location. ${ }^{18}$ Yet inclusion of non-localized 
disease poses a confounding factor since metastatic sites display heterogeneity from the primary tumor and is oftentimes highly anaplastic. ${ }^{19}$

Several studies have attempted to study the impact of location on outcomes in UTUC following radical surgery, but included patients with positive nodes and who received

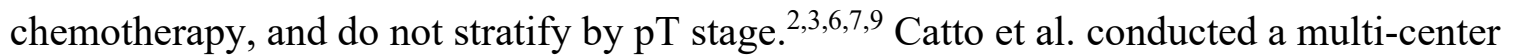
retrospective analysis and reported that for muscle invasive disease, location was not a predictor of mortality on univariable analysis; multivariable analysis was not included. ${ }^{2}$ Though not reaching statistical significance, both Favaretto et al. and Williams et al. published a KaplanMeier curve showing that overall survival for RPUC and UUC diverge, with RPUC having improved survival. ${ }^{3,9}$ Moussa et al. found no difference in overall survival for pT2 or greater between bladder and UTUC treated with radical surgery, but did not substratify UUC and RPUC in their analysis. ${ }^{6}$ NCDB data from 1998-2011 suggests no increased risk of death for RPUC versus UUC following radical nephroureterectomy. However, all pT stages are included in their Cox analysis, and $47 \%$ of their cohort consisted of pT 1 tumors and lower, ${ }^{20}$ confounding comparison with our cohort.

UTUC and bladder carcinoma may represent phenotypically distinct diseases. The urothelium of the upper tract and bladder are derived from different germ layers, with the bladder deriving from endoderm and both ureter and renal pelvis deriving from mesoderm.

Heterogeneity in the urothelium is suggested when examining risk factors for urothelial carcinoma. In Lynch syndrome, disease in the upper tract is more common than in the bladder. ${ }^{21}$ While the incidence of bladder cancer is also increased, it is unclear whether this is from seeding or from an intrinsic defect from mutations of Lynch syndrome. Consider that aristolochic acid exposure is associated with increased incidence of UTUC but not bladder cancer. ${ }^{22}$ The mutational patterns between the UTUC and BUC have been shown to differ. ${ }^{11,12}$ Audenet et al. showed that UTUC was more frequently associated with alterations in FGFR3 and HRAS, whereas TP53, RB1, and ERBB2 were more frequently altered in BUC. ${ }^{13}$ In patients with a history of UTUC and subsequent bladder UC, the tumors were "always clonally related," suggesting downstream seeding. The role of estrogen receptors on urothelial carcinoma has yet to be defined, though expression has been shown in a few studies to predict survival. ${ }^{23,24,25}$ The clinical significance of this marker is relevant when considering that the gender disparity for BUC is much more pronounced than for UTUC. ${ }^{1}$

The greatest limitation of this study is the lack of cancer-specific survival variables in the database. We opted to use overall survival, and sought to control for confounders by including Charlson-Deyo scores, days to discharge, and 30-day readmission in our multivariable analysis as a proxy for overall health. We acknowledge that overall survival is not a perfect substitute, but it likely correlates with cancer-specific survival in patients with muscle-invasive cancer. The retrospective observational design of this study introduces several biases, including selection, misclassification, and non-standardization of variables. Additionally, the NCDB does not 
provide details about $\mathrm{pT}$ stage - reclassification of renal parenchymal invasion into pT2 may nullify the survival advantage of pT3 RPUC. While the NCDB boasts large numbers, a small proportion of data points are incomplete and have missing variables. Despite these limitations, we feel that our large study population across the US and long duration of follow up bolsters the generalizability of this study. To our knowledge, this is the largest national study comparing survival between bladder and upper tract disease.

\section{Conclusions}

UTUC and BUC were historically viewed as identical diseases in different locations. Past literature may not have shown differences because analyses were not stratified by pT stage or chemotherapy received. When stratifying by $\mathrm{pT}$ stage, and controlling for use of neo-adjuvant and adjuvant therapies, pT3 in the renal pelvis is associated with a lower risk of mortality. This may be a consequence of embryological and genetic differences between the upper tract and bladder. Conceivably, subclassification of pT3 to separate parenchymal and peri-pelvic fat invasion may also explain this finding ${ }^{26}$. While treatment strategies for bladder cancer continue to evolve, strategies for UTUC have lagged behind. With the advent of targeted therapies, dedicated prospective studies are necessary to validate use in UTUC, rather than treating it as a subset on BUC, as was historically done. 


\section{References}

1. Green DA, Rink M, Xylinas E, et al. Urothelial carcinoma of the bladder and the upper tract: disparate twins. J Urol 2013;189(4):1214-21.

2. Catto JWF, Yates DR, Rehman I, et al. Behavior of urothelial carcinoma with respect to anatomical location. J Urol 2007;177(5):1715-20.

3. Favaretto RL, Shariat SF, Chade DC, et al. The effect of tumor location on prognosis in patients treated with radical nephroureterectomy at Memorial Sloan-Kettering Cancer Center. Eur Urol 2010;58(4):574-80.

4. Lughezzani G, Burger M, Margulis V, et al. Prognostic factors in upper urinary tract urothelial carcinomas: a comprehensive review of the current literature. Eur Urol 2012;62(1):100-14.

5. Isbarn $\mathrm{H}$, Jeldres $\mathrm{C}$, Shariat $\mathrm{SF}$, et al. Location of the primary tumor is not an independent predictor of cancer specific mortality in patients with upper urinary tract urothelial carcinoma. J Urol 2009;182(5):2177-81.

6. Moussa S, Yafi FA, El-Hakim A, et al. Outcome of surgical treatment of patients with upper versus lower urinary tract urothelial carcinoma: stage-by-stage comparison. Urol Int 2010;84(1):50-5.

7. Raman JD, Ng CK, Scherr DS, et al. Impact of tumor location on prognosis for patients with upper tract urothelial carcinoma managed by radical nephroureterectomy. Eur Urol 2010;57(6):1072-9.

8. Rink M, Ehdaie B, Cha EK, et al. Stage-specific impact of tumor location on oncologic outcomes in patients with upper and lower tract urothelial carcinoma following radical surgery. Eur Urol 2012;62(4):677-84.

9. Williams AK, Kassouf W, Chin J, et al. Multifocality rather than tumor location is a prognostic factor in upper tract urothelial carcinoma. Urol Oncol Semin Orig Investig 2013;31(7):1161-5.

10. Kim M, Jeong CW, Kwak C, et al. Are urothelial carcinomas of the upper urinary tract a distinct entity from urothelial carcinomas of the urinary bladder? Behavior of urothelial carcinoma after radical surgery with respect to anatomical location: a case control study. BMC Cancer 2015;15:149.

11. Moss TJ, Qi Y, Xi L, et al. Comprehensive genomic characterization of upper tract urothelial carcinoma. Eur Urol 2017;72(4):641-9.

12. Sfakianos JP, Cha EK, Iyer G, et al. Genomic characterization of upper tract urothelial carcinoma. Eur Urol 2015;68(6):970-7.

13. Audenet F, Isharwal S, Cha EK, et al. Clonal relatedness and mutational differences between upper tract and bladder urothelial carcinoma. Clin Cancer Res 2019;25(3):96776.

14. Roupret M, Babjuk M, Comperat E, et al. European Association of Urology Guidelines on Upper Urinary Tract Urothelial Carcinoma: 2017 Update. Eur Urol 2018;73(1):11122.

15. Park J, Park S, Song C, et al. Peripelvic/periureteral fat invasion is independently associated with worse prognosis in pT3 upper tract urothelial carcinoma. World J Urol 2014;32(1):157-63. 
16. Ouzzane A, Colin P, Xylinas E, et al. Ureteral and multifocal tumours have worse prognosis than renal pelvic tumours in urothelial carcinoma of the upper urinary tract treated by nephroureterectomy. Eur Urol 2011;60(6):1258-65.

17. Akdogan B, Dogan HS, Eskicorapci SY, et al. Prognostic significance of bladder tumor history and tumor location in upper tract transitional cell carcinoma. J Urol 2006;176(1):48-52.

18. Moschini M, Shariat SF, Rouprêt M, et al. Impact of primary tumor location on survival from the european organization for the research and treatment of cancer advanced urothelial cancer studies. J Urol 2018;199(5):1149-57.

19. Fidler IJ. Tumor heterogeneity and the biology of cancer invasion and metastasis. Cancer Research 1978; 38:2651-60.

20. Joshi SS, Quast LL, Chang SS, Patel SG. Effects of tumor size and location on survival in upper tract urothelial carcinoma after nephroureterectomy. Indian J Urol 2018;34(1):6873.

21. Win AK, Lindor NM, Young JP, et al. Risks of primary extracolonic cancers following colorectal cancer in lynch syndrome. J Natl Cancer Inst 2012;104(18):1363-72.

22. Petkovic SD. Epidemiology and treatment of renal pelvic and ureteral tumors. J Urol 1975;114(6):858-65.

23. Bolenz C, Lotan Y, Ashfaq R, Shariat SF. Estrogen and progesterone hormonal receptor expression in urothelial carcinoma of the bladder. Eur Urol 2009;56(6):1093-5.

24. Han B, Cui D, Jing Y, et al. Estrogen receptor beta (ERbeta) is a novel prognostic marker of recurrence survival in non-muscle-invasive bladder cancer potentially by inhibiting cadherin switch. World J Urol 2012;30(6):861-7.

25. Luo HL, Sung MT, Tsai EM, et al. Expression of estrogen receptor beta predicts oncologic outcome of pt 3 upper urinary tract urothelial carcinoma better than aggressive pathological features. Sci Rep 2016;6:24263.

26. Shariat SF, Zigeuner R, Rink M, et al. Subclassification of pT3 urothelial carcinoma of the renal pelvicalyceal system is associated with recurrence-free and cancer-specific survival: proposal for a revision of the current TNM classification. Eur Urol 2012;62(2):224-31. 


\section{Figures and Tables}

Fig. 1. Consort diagram. NCDB: National Cancer Database.

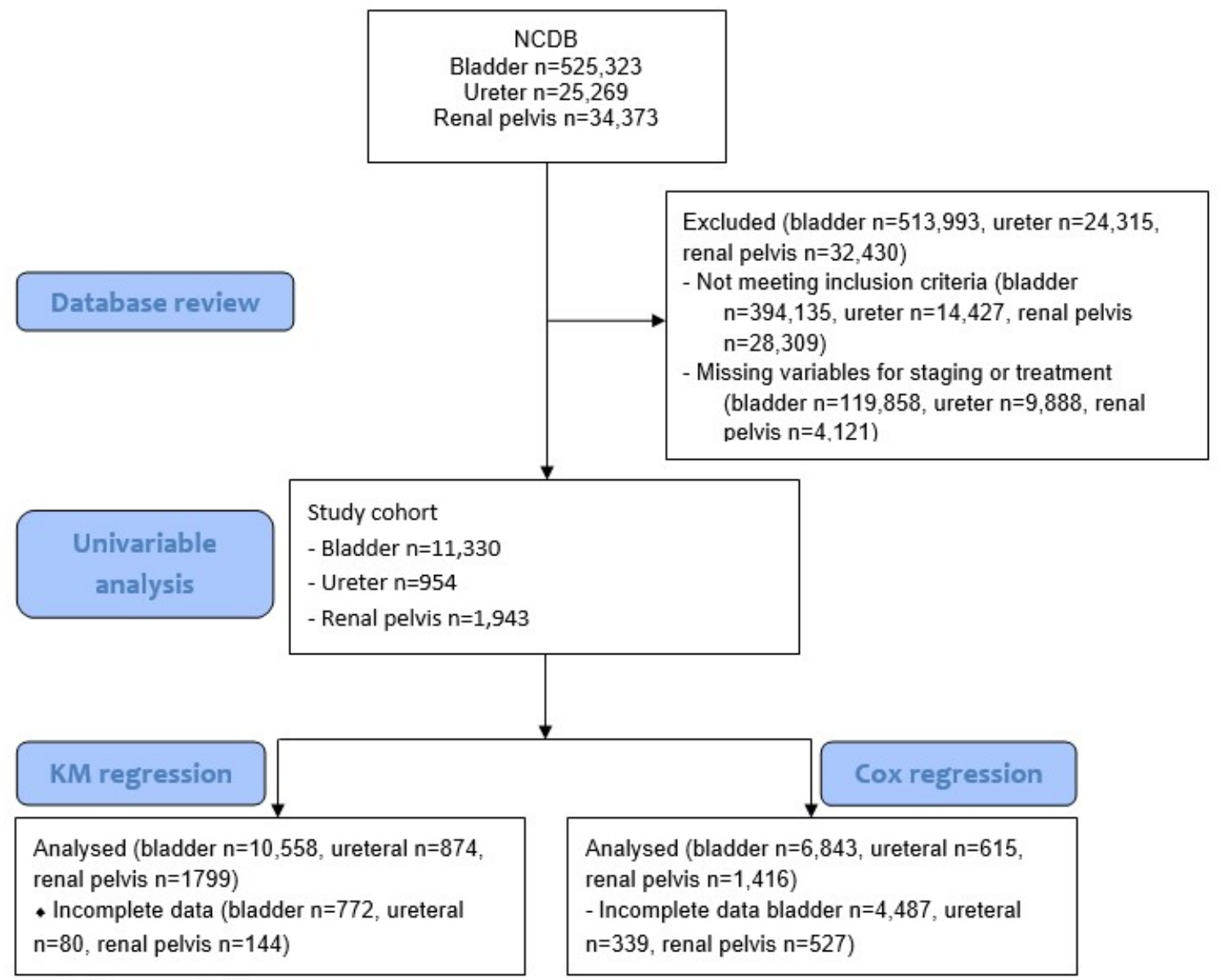


Fig. 2. Kaplan-Meier curve for overall survival of all patients, stratified by location. Bladder vs. ureteral $p<0.001$; bladder vs. renal pelvis $p=0.087$; ureteral vs. renal pelvis $p=0.028$.

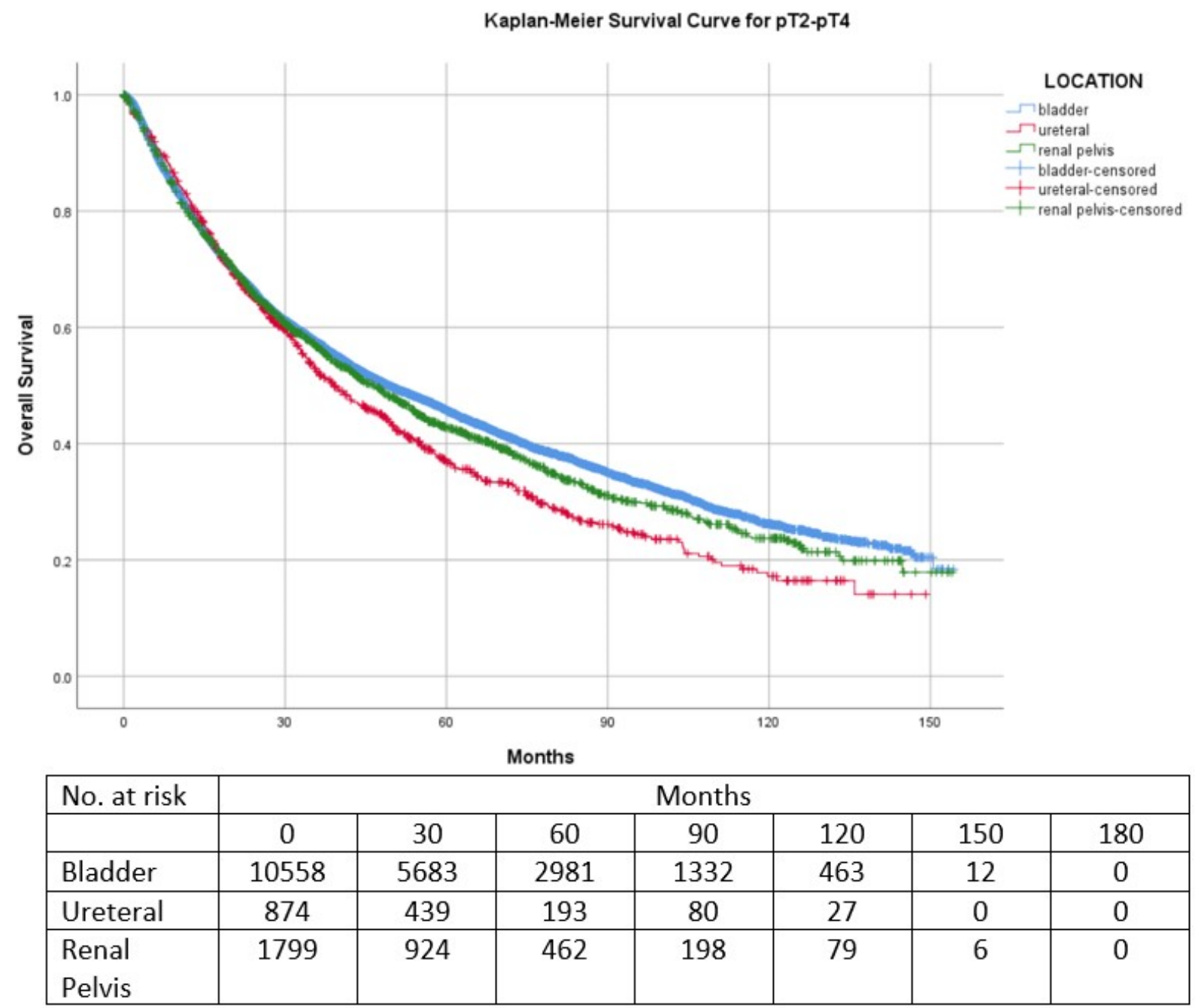


Fig. 3. Kaplan-Meier curves for overall survival stratified by pT stage.

(A) pT2: bladder vs. ureteral $\mathrm{p}=0.007$; bladder vs. renal pelvis $\mathrm{p}=0.531$; ureteral vs. renal pelvis $\mathrm{p}=0.014$.

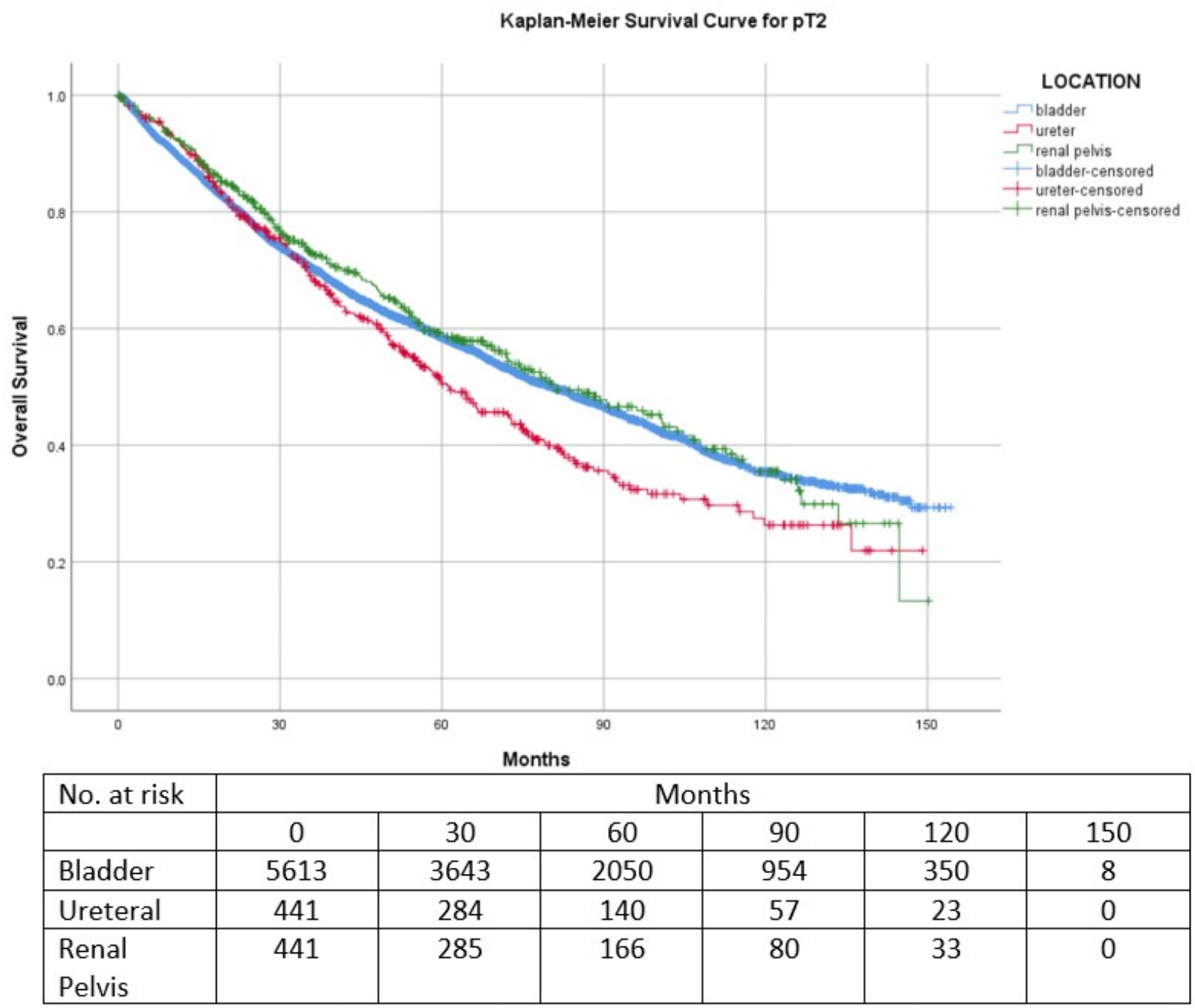


(B) $\mathrm{pT}$ 3: bladder vs. ureteral $\mathrm{p}=0.003$; bladder vs. renal pelvis $\mathrm{p}<0.001$; ureteral vs. renal pelvis $\mathrm{p}<0.001$.

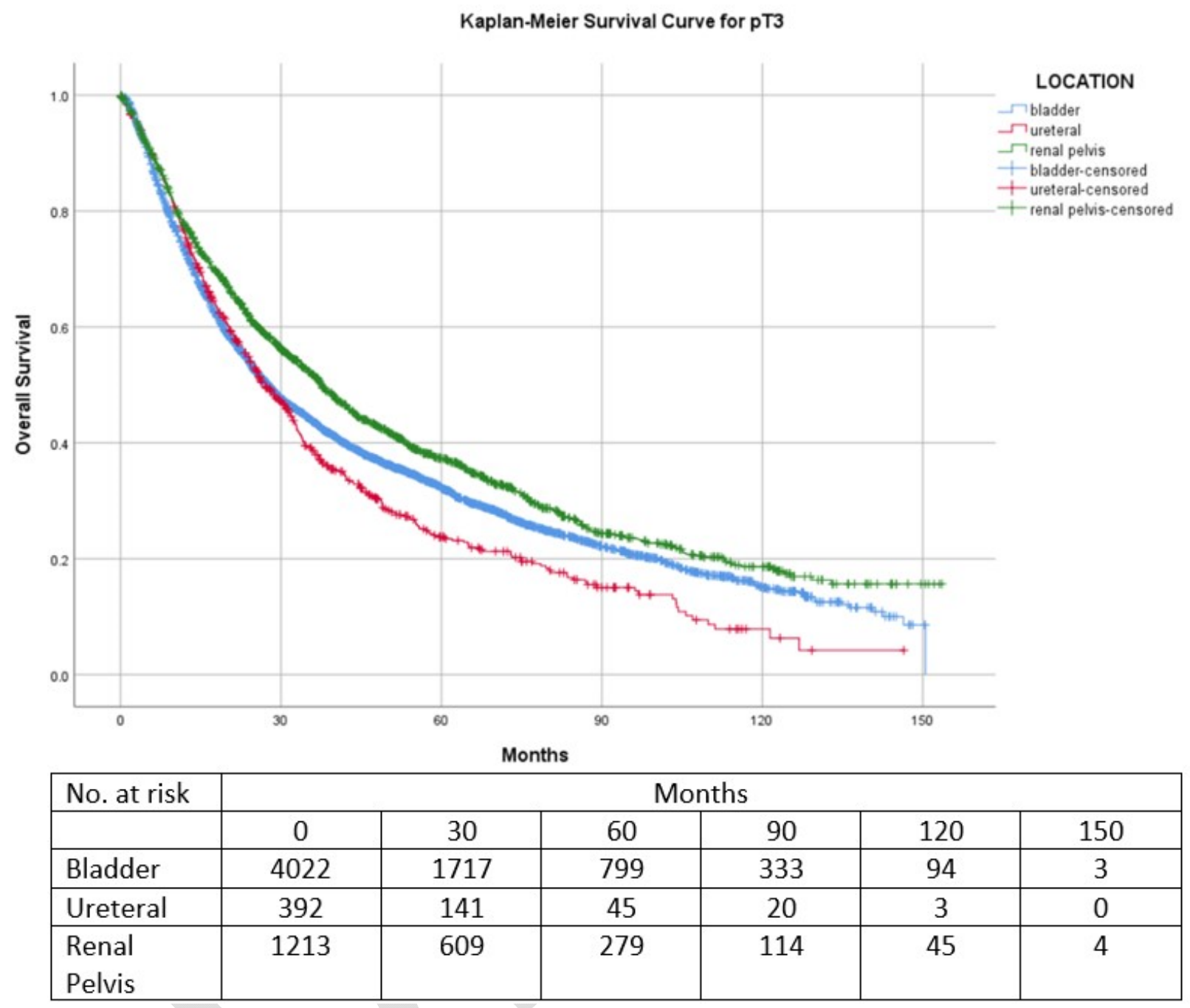


(C) pT4: bladder vs. ureteral $\mathrm{p}=0.847$; bladder vs. renal pelvis $\mathrm{p}<0.001$; ureteral vs. renal pelvis $\mathrm{p}=0.102$.

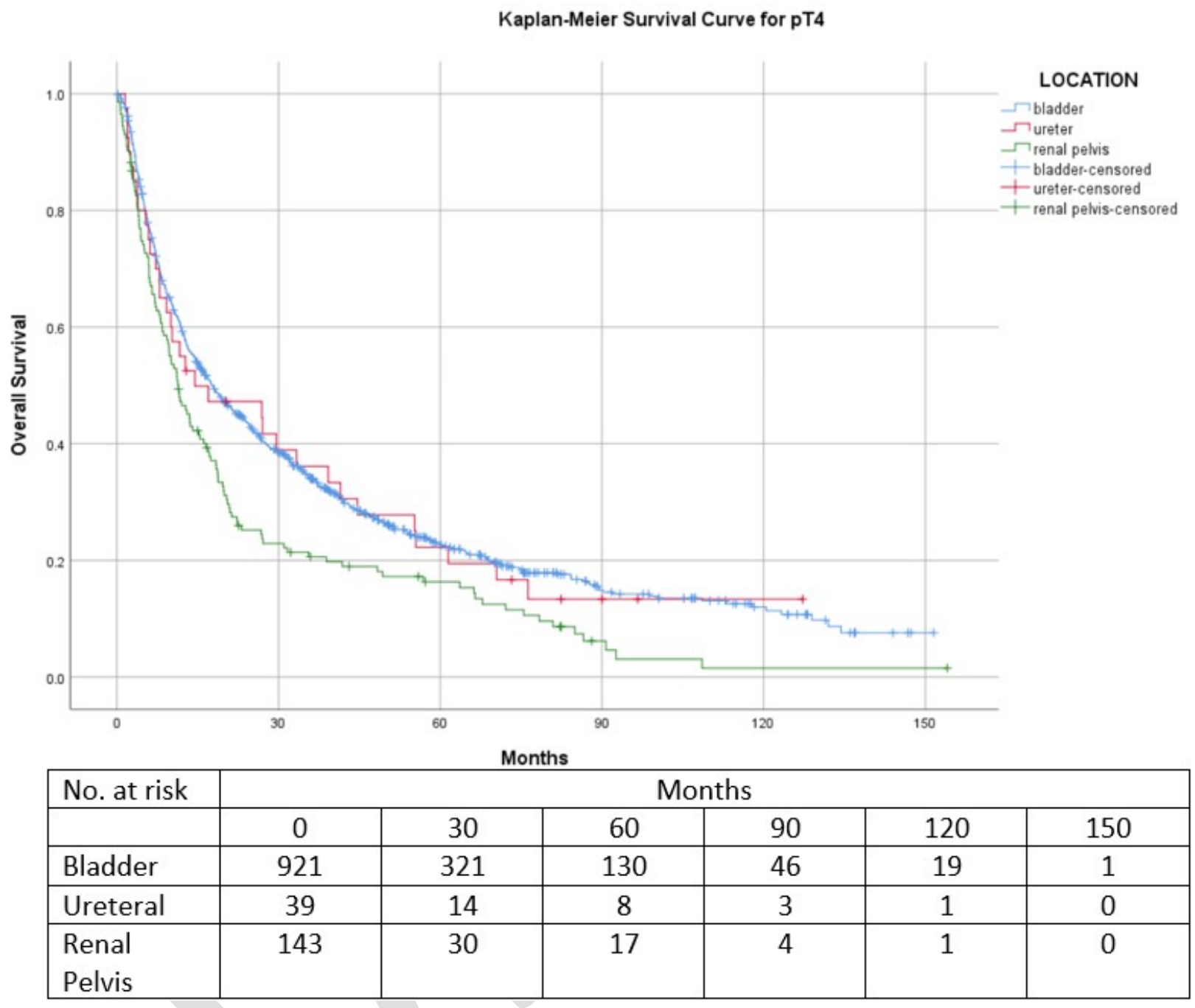




\begin{tabular}{|c|c|c|c|c|}
\hline & $\begin{array}{c}\text { Bladder } \\
(n=11330)\end{array}$ & $\begin{array}{c}\text { Ureter } \\
(n=954)\end{array}$ & $\begin{array}{c}\text { Renal pelvis } \\
(\mathrm{n}=1943)\end{array}$ & $\mathbf{p}$ \\
\hline Age (years) (median, IQR) & $70(62-77)$ & $74(66-81)$ & $74(65-81)$ & $<0.001$ \\
\hline Gender $(\%)$ & & & & $<0.001$ \\
\hline Male & $8603(75.9)$ & $539(56.5)$ & $1072(55.2)$ & \\
\hline Female & $2727(24.1)$ & $415(43.5)$ & $871(44.8)$ & \\
\hline Race $(\%)$ & & & & $<0.001$ \\
\hline White & $10353(92.3)$ & $872(92.4)$ & $1780(92.7)$ & \\
\hline Black & $631(5.6)$ & $35(3.7)$ & $84(4.4)$ & \\
\hline Other & $183(1.6)$ & $32(3.4)$ & $47(2.4)$ & \\
\hline Unknown & $52(0.5)$ & $5(0.5)$ & $10(0.5)$ & \\
\hline Median income quartile (\%) & & & & 0.001 \\
\hline Less than $\$ 38000$ & $1931(17.3)$ & $182(19.3)$ & $299(15.7)$ & \\
\hline$\$ 38000-47999$ & $2963(26.6)$ & $231(24.5)$ & $464(24.3)$ & \\
\hline$\$ 48000-62999$ & $3125(28)$ & $257(27.2)$ & $516(27)$ & \\
\hline Greater than $\$ 63000$ & $3134(28.1)$ & $274(29)$ & $629(33)$ & \\
\hline Year of diagnosis $(\%)$ & & +2 & & 0.032 \\
\hline $2004-2006$ & $3388(29.9)$ & $286(30)$ & $572(29.4)$ & \\
\hline $2007-2009$ & $2787(24.6)$ & $233(24.4)$ & $489(25.2)$ & \\
\hline $2010-2012$ & $2741(24.2)$ & $209(21.9)$ & $417(21.5)$ & \\
\hline $2013-2015$ & $2414(21.3)$ & $226(23.7)$ & $465(23.9)$ & \\
\hline Facility type (\%) & & & & $<0.001$ \\
\hline Community cancer program & $752(6.7)$ & $85(8.9)$ & $147(7.6)$ & \\
\hline $\begin{array}{l}\text { Comprehensive community } \\
\text { cancer }\end{array}$ & $4099(36.3)$ & $419(44)$ & $812(42.1)$ & \\
\hline Academic program & $5424(48.1)$ & $355(37.3)$ & $759(39.3)$ & \\
\hline $\begin{array}{l}\text { Integrated network cancer } \\
\text { program }\end{array}$ & $1011(9)$ & $94(9.9)$ & $212(11)$ & \\
\hline $\begin{array}{l}\text { Distance to facility of } \\
\text { diagnosis (miles) (median, } \\
\text { IQR) }\end{array}$ & $14.9(5.8-42.9)$ & $11.2(4.7-31.4)$ & $11.1(4.5-29.8)$ & $<0.001$ \\
\hline Charlson/Deyo score $(\%)$ & & & & 0.605 \\
\hline 0 & $7685(67.8)$ & $626(65.6)$ & $1305(67.2)$ & \\
\hline 1 & $2689(23.7)$ & $248(26)$ & $470(24.2)$ & \\
\hline 2 or more & $956(8.4)$ & $80(8.4)$ & $168(8.6)$ & \\
\hline T stage $(\%)$ & & & & $<0.001$ \\
\hline pT2 & $6002(53)$ & $472(49.5)$ & $474(24.4)$ & \\
\hline pT3 & $4331(38.2)$ & $440(46.1)$ & $1316(67.7)$ & \\
\hline pT4 & $997(8.8)$ & $42(4.4)$ & $153(7.9)$ & \\
\hline
\end{tabular}




\begin{tabular}{|l|c|c|c|c|}
\hline ICD-O-3 tumor grade (\%) & & & & $<0.001$ \\
\hline 1 & $95(0.9)$ & $36(4.2)$ & $88(5.1)$ & \\
\hline 2 & $389(3.8)$ & $94(11.1)$ & $217(12.6)$ & \\
\hline 3 & $5629(54.5)$ & $439(51.6)$ & $843(48.8)$ & \\
\hline 4 & $4212(40.8)$ & $281(33.1)$ & $580(33.6)$ & \\
\hline $\begin{array}{l}\text { Tumor size (cm) (median, } \\
\text { IQR) }\end{array}$ & $3.7(2.5-5.2)$ & $3(2-5)$ & $4(2.8-5.5)$ & $<0.001$ \\
\hline Bilateral disease (\%) & $\mathrm{n} / \mathrm{a}$ & $0(0)$ & $1(0.1)$ & $<0.001$ \\
\hline Positive surgical margins (\%) & $699(6.4)$ & $148(15.8)$ & $117(6.1)$ & $<0.001$ \\
\hline Lymphadenectomy (\%) & $10,421(92)$ & $411(43.1)$ & $697(35.9)$ & $<0.001$ \\
\hline $\begin{array}{l}\text { Nodes examined (median, } \\
\text { IQR) }\end{array}$ & $10(4-17)$ & $0(0-2)$ & $0(1-6)$ & $<0.001$ \\
\hline $\begin{array}{l}\text { Days to definitive surgery } \\
\text { (median, IQR) }\end{array}$ & $47(29-70)$ & $26(3.5-46)$ & $21(0-44)$ & $<0.001$ \\
\hline $\begin{array}{l}\text { Days to discharge (median, } \\
\text { IQR) }\end{array}$ & $8(6-11)$ & $5(3-7)$ & $4(3-6)$ & $<0.001$ \\
\hline $\begin{array}{l}\text { Readmission within 30 days } \\
\text { of surgery (\%) }\end{array}$ & $1219(11)$ & $46(4.9)$ & $97(5.1)$ & $<0.001$ \\
\hline $\begin{array}{l}\text { Mortality within 30 days of } \\
\text { surgery (\%) }\end{array}$ & $355(3.4)$ & $26(3)$ & $48(2.7)$ & 0.105 \\
\hline $\begin{array}{l}\text { Mortality within 90 days of } \\
\text { surgery (\%) }\end{array}$ & $797(7.5)$ & $49(5.6)$ & $113(6.3)$ & 0.023 \\
\hline
\end{tabular}

Univariable anaylysis was completed with Chi-squared tests for categorical variables and Kruskal-Wallis test for continuous variables. IQR: interquartile range;

Table 2A. Multivariable Cox regression examining predictors of mortality for patients
with pT2 disease
\begin{tabular}{|c|c|c|c|}
\hline & Hazard ratio & $\mathbf{9 5 \%}$ CI & p \\
\hline Location & & & \\
\hline Bladder & Ref & & 0.856 \\
\hline Ureteral & 0.99 & $0.85-1.14$ & 0.125 \\
\hline Renal pelvis & 0.89 & $0.76-1.04$ & $<0.001^{*}$ \\
\hline Age (continuous) & 1.04 & $1.03-1.04$ & \\
\hline Gender & & & 0.176 \\
\hline Male & Ref & & \\
\hline Female & 0.94 & $0.85-1.03$ & \\
\hline Charlson/Deyo score & & & $<0.001^{*}$ \\
\hline 0 & Ref & & $<0.001^{*}$ \\
\hline 1 & 1.26 & $1.14-1.39$ & \\
\hline 2 or more & 1.66 & $1.43-1.92$ & \\
\hline ICD-O-3 tumor grade & & & \\
\hline 1 & Ref & & \\
\hline
\end{tabular}




\begin{tabular}{|l|c|c|c|}
\hline \multicolumn{1}{|c|}{} & 0.91 & $0.66-1.26$ & 0.559 \\
\hline 3 & 1.20 & $0.90-1.59$ & 0.213 \\
\hline 4 & 1.18 & $0.89-1.58$ & 0.255 \\
\hline Tumor size (continuous) & 1.001 & $1.000-1.002$ & $0.004^{*}$ \\
\hline Positive surgical margins & 1.55 & $1.27-1.88$ & $<0.001^{*}$ \\
\hline Nodes examined (continuous) & 0.99 & $0.986-0.995$ & $<0.001^{*}$ \\
\hline Days to discharge (continuous) & 1.01 & $1.004-1.010$ & $<0.001^{*}$ \\
\hline $\begin{array}{l}\text { Readmission within 30 days of } \\
\text { surgery }\end{array}$ & 1.11 & $0.97-1.27$ & 0.122 \\
\hline
\end{tabular}

*Statistically significant. CI: confidence interval.

\begin{tabular}{|c|c|c|c|}
\hline & Hazard ratio & 95\% CI & $\mathbf{p}$ \\
\hline \multicolumn{4}{|l|}{ Location } \\
\hline Bladder & Ref & 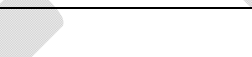 & 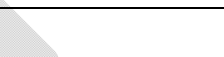 \\
\hline Ureteral & 0.98 & $0.86-1.12$ & 0.793 \\
\hline Renal pelvis & 0.80 & $0.72-0.88$ & $<0.001^{*}$ \\
\hline Age (continuous) & 1.03 & $1.02-1.03$ & $<0.001^{*}$ \\
\hline \multicolumn{4}{|l|}{ Gender } \\
\hline Male & Ref & +2 & \\
\hline Female & 0.89 & $0.82-0.96$ & $0.002^{*}$ \\
\hline \multicolumn{4}{|l|}{ Charlson/Deyo score } \\
\hline 0 & Ref & & \\
\hline 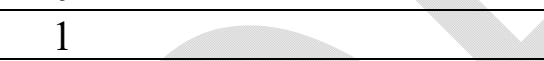 & 1.24 & $1.14-1.34$ & $<0.001^{*}$ \\
\hline 2 or more & 1.59 & $1.42-1.78$ & $<0.001^{*}$ \\
\hline \multicolumn{4}{|c|}{ ICD-O-3 tumor grade } \\
\hline 1 & Ref & & \\
\hline 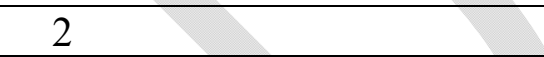 & 1.20 & $0.86-1.68$ & 0.286 \\
\hline+2 & 1.54 & $1.13-2.09$ & $0.006^{*}$ \\
\hline 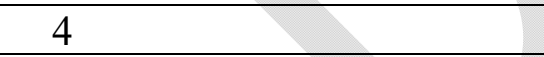 & 1.45 & $1.06-1.97$ & $0.019^{*}$ \\
\hline Tumor size (continuous) & 1.003 & $1.003-1.004$ & $<0.001^{*}$ \\
\hline Positive surgical margins & 1.66 & $1.48-1.86$ & $<0.001^{*}$ \\
\hline Nodes examined (continuous) & 0.99 & $0.98-0.99$ & $<0.001^{*}$ \\
\hline Days to discharge (continuous) & 1.01 & $1.01-1.01$ & $<0.001^{*}$ \\
\hline $\begin{array}{l}\text { Readmission within } 30 \text { days of } \\
\text { surgery }\end{array}$ & 1.02 & $0.91-1.15$ & 0.724 \\
\hline
\end{tabular}

${ }^{*}$ Statistically significant. CI: confidence interval. 


\begin{tabular}{|c|c|c|c|}
\hline & Hazard ratio & $95 \% \mathrm{CI}$ & $\mathbf{p}$ \\
\hline \multicolumn{4}{|l|}{ Location } \\
\hline Bladder & Ref & & \\
\hline Ureteral & 0.98 & $0.66-1.43$ & 0.899 \\
\hline Renal pelvis & 1.19 & $0.96-1.46$ & 0.108 \\
\hline Age (continuous) & 1.02 & $1.01-1.03$ & $<0.001^{*}$ \\
\hline \multicolumn{4}{|l|}{ Gender } \\
\hline Male & Ref & +2 & \\
\hline Female & 1.19 & $1.00-1.41$ & 0.056 \\
\hline \multicolumn{4}{|l|}{ Charlson/Deyo score } \\
\hline 0 & Ref & +2 & \\
\hline 1 & 1.01 & $0.85-1.21$ & 0.898 \\
\hline 2 or more & 1.43 & $1.14-1.81$ & $0.002^{*}$ \\
\hline \multicolumn{4}{|l|}{ ICD-O-3 tumor grade } \\
\hline 1 & Ref & +2 & \\
\hline 2 & 1.51 & $0.56-4.08$ & 0.414 \\
\hline 3 & 2.20 & $0.89-5.44$ & 0.089 \\
\hline 4 & 1.83 & $0.74-4.54$ & 0.193 \\
\hline Tumor size (continuous) & 1.001 & $1.000-1.001$ & 0.100 \\
\hline Positive surgical margins & 1.71 & $1.45-2.02$ & $<0.001^{*}$ \\
\hline Nodes examined (continuous) & 0.99 & $0.985-0.992$ & $0.034^{*}$ \\
\hline Days to discharge (continuous) & 1.01 & $1.01-1.02$ & $<0.001^{*}$ \\
\hline $\begin{array}{l}\text { Readmission within } 30 \text { days of } \\
\text { surgery }\end{array}$ & 1.25 & $0.99-1.58$ & 0.062 \\
\hline
\end{tabular}

*Statistically significant. CI: confidence interval.

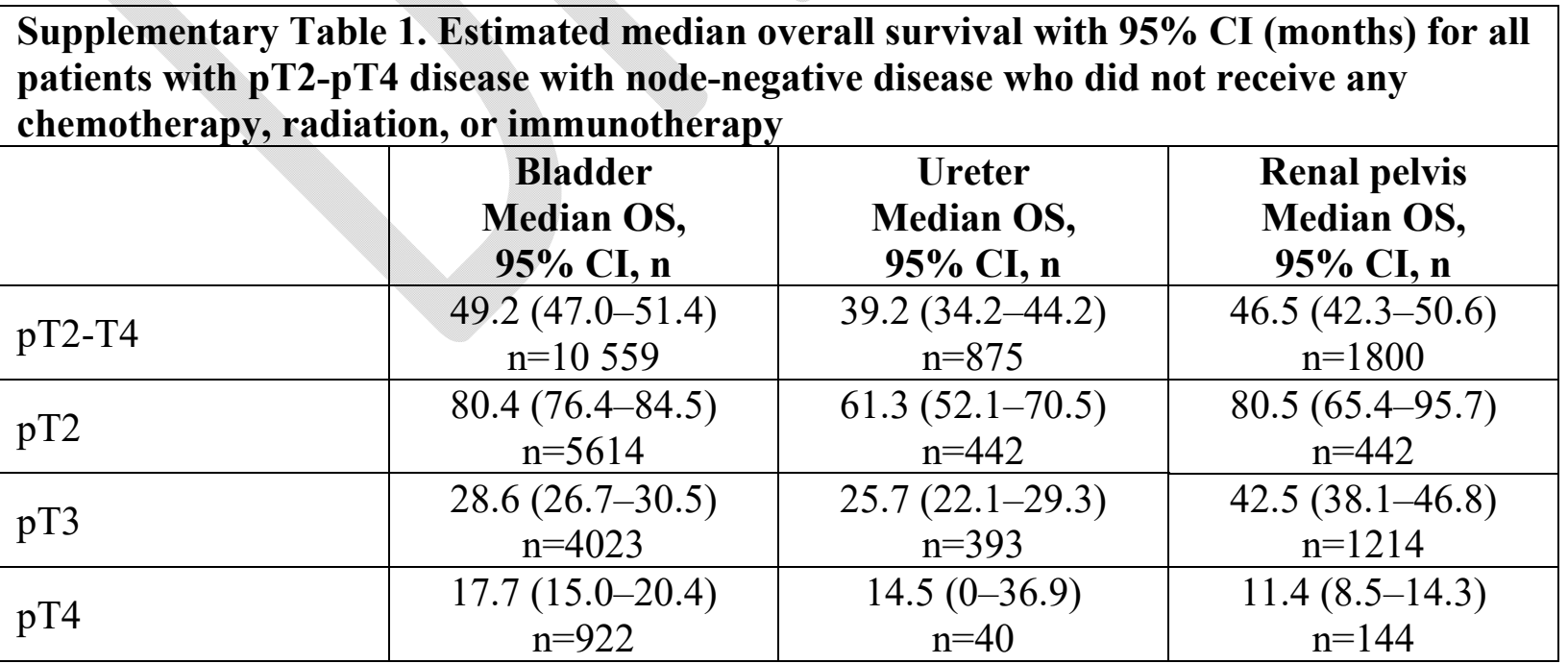

The $\mathrm{n}$ for each strata subset is included as well. CI: confidence interval; OS: overall survival. 